\title{
Path integrals in fluctuating markets
}

\author{
Frédéric D.R. Bonnet ${ }^{a, b}$, Andrew Allison ${ }^{a, b}$ and Derek Abbott ${ }^{a, b}$ \\ ${ }^{a}$ Department of Electrical and Electronic Engineering, The University of Adelaide, S.A. 5005, \\ Australia. \\ ${ }^{b}$ Centre for Biomedical Engineering, The University of Adelaide, S.A. 5005, Australia.
}

\begin{abstract}
In this short note we propose an approach for calculating option prices in financial markets in the framework of path integrals. We review various techniques from engineering and physics applied to the theory of financial risks. We explore how the path integral methods may be used to study financial markets quantitatively and we also suggest a method in calculating transition probabilities for option pricing using real data in that framework.
\end{abstract}

Keywords: Stock market, path integrals, econoengineering, financial risks, Black-Scholes equation, quantum field theory, stochastic signal processes.

\section{INTRODUCTION}

Economics and the idea of trading goods can be found to have roots as far as the ancient times at the cradle of all human civilization. This concept and the resulting methods have evolved into a complicated system that involves billions of simultaneous transactions in the world. Presently all transactions are computerized- this revolution began in the 1980's when electronic trading, already a part of the environment of the major stock exchange, was adapted to the foreign exchange market. As a result we have today a huge amount of electronically stored financial data ready to be analyzed. The average time delay between two records can be as short as a few seconds. Therefore one could use this data to study short or long term effects.

Economists and mathematicians are known to draw models to help them predict parameters or trends in financial markets. ${ }^{1}$ However, in the recent years more engineers and physicists are becoming involved in the analysis of economic systems and are bringing new concepts and tools to some long standing problems in financial markets research. The standard approach used by physicists is to apply techniques used in statistical mechanics that they have been able to apply to get some fresh insights into the dynamics of the financial markets. For example attempts have been made to study the dynamics using a maximal entropy approach ${ }^{2}$ and in a paper by P.Buchen and M. Kelly, ${ }^{3}$ it has been shown that the data they used was not sufficient to uniquely determine distribution of the assets. Alternatively a modified Ising model to study stochastic resonance and model financial crashes ${ }^{4}$ has been used. Stochastic Differential Equations (SDE) have also been exploited in the evaluation of option pricing, ${ }^{5-7}$ and has been found to be successful in developing a theory of non-Gaussian option pricing which allows for closed form solutions for European options, which are such that can be exercised exclusively on a fixed day of expiration and not before (as in the case of American options), ${ }^{8,9}$ their approach uses stochastic processes with statistical feedback ${ }^{10}$ as a model for stock prices. Such processes were developed within the Tsallis generalized thermostatistics. ${ }^{11}$

Options are ancient financial tools, they are used for speculative purposes or for hedging major market transactions against unexpected changes in the market environment. These can produce large fluctuations in the price of the assets, and options are intended to prevent the destruction of large amounts of capital. Historically, ancient Romans, Grecians and Phoenicians traded options against outgoing cargo from their local sea ports. In more recent times option pricing techniques have their roots in early work by C. Castelli who published in 1877 a book entitled The Theory of Option in Stocks and Shares. The earliest known analytical valuation for the option

Further author information: (Send correspondence to F.D.R. Bonnet.)

F.D.R. Bonnet.: E-mail: fbonnet@eleceng.adelaide.edu.au, Telephone: +61 883036296

A. Allison: E-mail: aallison@eleceng.adelaide.edu.au, Telephone: +61 88303-5283

D. Abbott.: E-mail: dabbott@eleceng.adelaide.edu.au, Telephone: +61 883035748

Noise in Complex Systems and Stochastic Dynamics II, edited by Zoltán Gingl, 
was offered by Louis Bachelier in his dissertation at La Sorbonne. ${ }^{12}$ Louis Bachelier discovered the treatment of stochastic phenomena five years before Einstein related but much more famous work on Brownian motion ${ }^{13}$ and twenty three years before Wiener mathematical development, ${ }^{14}$ which led the discovery in 1973 by Fisher Black and Merton Schloes of what is famously called Black-Scholes model. ${ }^{15}$ Present day options are contracts between two parties where each party has the right, but is not obligated, to buy or sell some assets. This right has a value which must be purchased at a given price. This price usually depends on the value of the asset in question, hence the name of derivative security by pricing and risk management of such financial instruments is a major focus of financial market research.

In the Black-Scholes option pricing model, one normally assumes that the system follows a geometric Brownian motion for some underlying asset, which is described by an Ito stochastic differential equation (SDE). The disadvantage of the Black-Scholes Model is that it is based on several assumptions, namely:

1) Markets are efficient, 2) the returns are normally distributed, 3) no commissions are charged, 4) interest rates remain constant and known, 5) the stock pays no dividend during the options life dividends, 6) European exercise terms are used.

Since 1973 the original Black-Scholes Option Pricing Model has been improved and extended considerably. R. Merton ${ }^{16}$ included the effect of dividends, three years later. J. Ingersoll relaxed the assumption of no taxes or transaction costs, and Merton removed the restriction of constant interest rates. Currently, we are now in a position of being able to determine the values of a large variety of different options. However many challenges remain in particular for options with non-standard payoff features (such as various barrier offers many of those features which are designed to reduce options cost by removing protection from risk, considered highly volatility or that the purchase may be willing to bear) and multi-factor options (i.e., that depends on several underlying assets), these are the options that are commonly traded in real markets and allow anticipated exercise and/or depend on the history of the underlying asset.

Thus the challenge remains to develop more efficient computational tools that financial analyst can apply to the complex ever growing array of more complex derivative instruments being developed by financial markets. One promising approach involves the use of path integrals that have been well developed by physicists (in fact the most important use of path integrals in financial markets is made in the determination of a fair price of financial derivatives in particular options). The relevance of path integrals to this field was first recognized in 1988 by the theoretical physicist Dash who wrote two unpublished papers on the subject entitled Path Integrals and Option Pricing I and II. Since then many theoretical physicists have entered the field and papers on the subject have begun to appear on the Los Alamos archive. ${ }^{17-21}$

Path integrals were first introduced by Wiener ${ }^{22,23}$ and then futher developed by Feynman $(1948)^{24}$ to perform calculations in quantum electrodynamics (QED). The method has now become a very important one as well as representing a very powerful tool for elementary particle physicists who study the theory of strong interactions Quantum Chromodynamics (QCD), where it is possible to use Lattice Gauge Theory to study the fundamental particles of nature that make up the entire universe, such as quarks and gluons. ${ }^{25-29}$ In that theory a powerful technique, using cutting edge scientific computing, ${ }^{30-32}$ is used to approximate the path integral and calculate both gauge dependent and gauge independent quantities and observables. ${ }^{33}$ These techniques may be of use in the future for option pricing ${ }^{34}$ where one could formulate a lattice gauge theory for path integrals once a better understanding of the path integral for option pricing is known, which ultimately leads to Monte Carlo simulation of the stock market. Engineers do use path integrals, for example, when studying radio frequencing propagation. ${ }^{35}$ Applications of path integral techniques ${ }^{36}$ to financial markets have also recently started to appear in the literature: for example $\mathrm{e}^{37-39}$ and the references therein. The advantage of path integrals when calculating an observable is that it takes into consideration the fluctuations associated with it and gives an alternative approach to the Black-Scholes-Merton model (BSM). Moreover in the most simple case of a so-called European option, the BSM equation can be explicitly solved to obtain an analytical formula for the price of the option, but when we consider the more complex financial derivatives discussed earlier, the BSM formula fails to give an analytical result. Appropriate numerical procedures have been developed in the literature to price exotic financial derivatives with path-dependent features as discussed in Refs. ${ }^{40,41}$ Moreover it is widely recognized that the simplicity of the popular Black-Scholes model, which relates derivatives prices to current stock prices and quantifies risk through a constant volatility parameter, is no longer sufficient to capture modern market 
phenomena especially since the 1987 crash. It has been observed that the real markets display high volatility as a stochastic process, the modelling of volatility as a stochastic process has been motivated a priory by empirical studies of stock prices returns in which estimated volatility is observed to exhibit random characteristics. ${ }^{42}$

The feature of the path integral technique that makes it useful for the option pricing situation is that it provides a very convenient way of tracking the evolution of the state of the system over time. In option pricing context the state of the system is the vector of prices of underlying assets usually stocks and bond. So if we know the state of the system at a particular initial time, it is then possible to determine the state of that system at a later time in the future. In general one may thus calculate some transition probability that is used to calculate an expectation value of some functional of a given stochastic process. In the option pricing context this means calculating the expectation (under the risk neutral measure) of the payoff over possible outcome for the underlying securities. For example for a European call option at the maturity time $T$, the quantity of interest would be $\max \left\{S_{T}-X, 0\right\}$, where $X$ is the strike price and $S_{T}$ is the price of an asset at the maturity time $T$.

An accurate method for calculating option prices in the path integral approach remains to be formulated. Path integrals are useful in this case because they involve a quantised theory, and all of the fluctuations arising from the system are taken into consideration and therefore provides a complete description of the system in question. Unlike a classical approach, each fluctuation is associated with a path, and each path is weighted according to some transition probability.

So far, various attempts have been made to calculate option price using path integrals, however up to now all of the formulations that have been published, lead to analytic exact solutions of the path integral due to the insertion of relatively simple probability transition functions. ${ }^{37-39,43-46}$ Our aim in this research program is to remove these simplifications thereby allowing application to the far more complex option pricing situation referred to earlier.

To achieve this goal, we will investigate numerical solutions to path integrals, as it will be explained in Section 5. Existing financial data will be used to formulate a numerical model for the transition probabilities, which will then be inserted as input data with a set of parameters into the path integral, hence this would lead to a more accurate calculation of the option price. This may be repeated iteratively.

The results obtained will then be compared to results obtained from the Black-Scholes equations for option pricing.

The advantages of this method are that, 1) real data will be used as input, 2) an automated numerical solution will arise and 3) the method will provide an approach that requires fewer assumptions in the construction of our model, as opposed to the Black-Scholes formulation of option pricing or in models presented by M. Rubinstein ${ }^{47,48}$ where they used various assumptions like a) underlying asset return follows a binomial process b) The binomial tree is recombining c) The ending nodal values are ordered from the lowest to highest.

The outline of this note first describes some of the concepts on the Black-Scholes equation, I describe the approach that will be taken.

\section{CONCEPTS AND THE BLACK \& SCHOLES FORMULA}

\subsection{Some Concepts}

In finance one usually deals with a special class of financial contracts called derivatives. A derivative is a financial product whose price depends upon the price of another financial product. These derivatives include, for example, forward contracts, futures, options and swaps. Here we are only going to focus on the first three of these.

A forward contract is when one of the parties agrees to buy for a given amount an asset at a specified price (called the forward price or the delivery price $K$ ) on a specified future date (the delivery date $T$ ). The other party agrees to sell the specified amount of the asset at the delivery price on the delivery date.

Futures are a forward contract traded on by exchange. The exchange is done through an exchange institution or a delivery house.

An option is a financial contract that gives the holder the right to exercise a given action (usually buying or selling) on an underlying asset of time $T$ and at a price $K$. The price $K$ is called the strike price and $T$ is called 
the expiration date, the exercise date or the date of maturity. There are two types of options, namely there is the Call option, which gives the holder the right to buy the underlying asset by a certain date for a certain price. Oppositely we have a Put option that is the same as the call option but instead of buying it is when selling.

There are a few different sorts of options and each of them carry a different name:

- American options: can be exercised at any time up to the expiration date.

- European option: can only be exercised on the expiration date itself *

- Exotic or Path dependent options: these options have values which depend on the history of an asset price not just its value on exercise. An example would be an option to purchase an asset for the arithmetic average value of that asset over the month before expiring.

- Barrier option: the option can either come into existence or become worthless if the underlying asset reaches some prescribed value before expiring.

- Asian option: the price depends on some form of average.

- Lookback options: the price depends on asset price maximum or minimum.

Swaps involve a private agreement between two parties to exchange cash flows at a certain times in the future according to some prearranged formula.

\subsection{Option pricing in idealized markets}

The idea is to find the rational and fair price $C(Y, t)$ of the option under consideration. Since the price $Y$ at each time step $t$ is a random variable, the value of the option at a time $t$ is a function of a random variable.

In 1973 Black and Scholes ${ }^{15,16}$ proposed the first reliable solution of the option-pricing problem. In their solution they assumed that the stock price $Y(t)$ can be described as an Ito process, namely a process defined by the stochastic differential equation $d Y=a(Y, t) d t+b(Y, t) d W$. In particular they assume that a stock price follows a geometric Brownian motion

$$
d Y=\mu Y d t+\sigma Y d W
$$

where $\mu$ is the expected return per unit time, $\sigma^{2}$ the variance per unit time, and $W$ is a Wiener process. That is $a=\mu Y$ and $b=\sigma Y$. This assumption implies that the changes in the logarithm of price are Gaussian distributed.

In that case, any function of $Y$ must be a solution of the partial differential equation obtained from a special case of Ito's lemma, Eq. (66), valid for a geometric Brownian motion ${ }^{49}$ (see Appendix A),

$$
d C=\left[\frac{\partial C}{\partial Y} \mu Y+\frac{\partial C}{\partial t}+\frac{1}{2} \frac{\partial^{2} C}{\partial Y^{2}} \sigma^{2} Y^{2}\right] d t+\frac{\partial C}{\partial Y} \sigma Y d W
$$

If we consider the portfolio of the holder is made up of number of shares $\Delta_{h}$ and the holder is selling one derivative of the stock at time $t$, where in the limit when $t$ becomes infinitesimal, $\Delta_{h}=\partial C / \partial Y$, the value of the portfolio is then

$$
\phi=\frac{\partial C}{\partial Y} Y-C
$$

which implies that the change in the value of the portfolio $\phi$ over a time interval $\Delta t$ is

$$
\Delta \phi=\frac{\partial C}{\partial Y} \Delta Y-\Delta C
$$

*Note that the terms "American" and "European" do not refer to the location of the option or the exchange. Some options trading on North American exchanges are European. 
Using Ito's lemma, we have

$$
\Delta C=\left[\frac{\partial C}{\partial Y} \mu Y+\frac{\partial C}{\partial t}+\frac{1}{2} \frac{\partial^{2} C}{\partial Y^{2}} \sigma^{2} Y^{2}\right] \Delta t+\frac{\partial C}{\partial Y} \sigma Y \Delta W .
$$

From Eq. (1), we have

$$
\Delta Y=\mu Y \Delta t+\sigma Y \Delta W
$$

Hence the change in $\phi$ may be rewritten and simplified to

$$
\Delta \phi=-\left[\frac{\partial C}{\partial t}+\frac{1}{2} \frac{\partial^{2} C}{\partial Y^{2}} \sigma^{2} Y^{2}\right] \Delta t .
$$

The Black and Scholes assumption that a stock price follows a geometric Brownian motion turns out to be crucial in deriving the rational price of an option. In fact without this assumption, $\Delta \phi$ could not be simplified as it is in Eq. (7). The second key assumption concerns the absence of arbitrage, which means that the change in the value of the portfolio $\Delta \phi$ must equal the gain obtained by investing the same amount of money in a riskless security that provides a return per unit of time $r$. Under the assumption that $r$ is constant,

$$
\Delta \phi=r \phi \Delta t .
$$

By equating Eqs. (7) and (8) we obtain

$$
r C=\frac{\partial C}{\partial t}+\frac{1}{2} \frac{\partial^{2} C}{\partial Y^{2}} \sigma^{2} Y^{2}+r Y \frac{\partial C}{\partial Y} .
$$

Eq. (9) is called the Black-Scholes partial differential equation. To obtain Eq. (9), no assumption about the specific kind of option has been made. This partial differential equation is valid for both call and put options. The appropriate $C(Y, t)$ for the chosen type of option will be obtained by selecting the appropriate boundary conditions, for example for a call option,

$$
C=\max \{Y-K, 0\} \quad \text { when } \quad t=T .
$$

\subsection{The Black-Scholes formula}

Black and Scholes ${ }^{15}$ solved the partial differential equation, Eq. (9) (known as the Black-Scholes partial differential equation) by making the following substitution

$$
C(Y, t)=\exp [r(t-T)] y\left(x, t^{\prime}\right)
$$

where in this case the variable $x$ is defined as

$$
x \equiv \frac{2}{\sigma^{2}}\left(r-\frac{1}{2} \sigma^{2}\right)\left[\ln \left(\frac{Y}{K}\right)-\left(r-\frac{1}{2} \sigma^{2}\right)(t-T)\right]
$$

and the variables $t^{\prime}$ defined as

$$
t^{\prime} \equiv-\frac{2}{\sigma^{2}}\left(r-\frac{1}{2} \sigma^{2}\right)(t-T) .
$$

With this substitution, the Black-Scholes partial differential equation reduces to a partial differential equation describing the heat-transfer in physical systems, namely

$$
\frac{\partial y\left(x, t^{\prime}\right)}{\partial t^{\prime}}=\frac{\partial^{2} y\left(x, t^{\prime}\right)}{\partial x^{2}}
$$

which can be solved analytically. Using Eq. 12 and 13, we find the solution takes the form of

$$
C(Y, t)=Y N\left(d_{1}\right)-K \exp [r(t-T)] N\left(d_{2}\right),
$$


where $N(x)$ is the cumulative density function for a Gaussian variable with zero mean and unit standard deviation,

$$
d_{1} \equiv \frac{\ln (Y / K)+\left(r+\sigma^{2} / 2\right)(T-t)}{\sigma \sqrt{T-t}}
$$

and

$$
d_{2} \equiv d_{1}-\sigma \sqrt{T-t} .
$$

The Black-Scholes model provides two important financial instruments. The first one is that it brings us an analytical solution for the rational price of a European option, secondly a trading strategy for building up a riskless portfolio. However the solution of the equation is only valid under the following assumptions:

1. the stock price follows Ito's stochastic process (see Appendix A for more details);

2. security trading is continuous;

3. there are no arbitrage opportunities;

4. selling of securities is possible at any time;

5. there are no transaction costs;

6. the market interest rate $r$ is constant; and

7. there are no dividends between $t=0$ and $t=T$.

Hence the Black-Scholes model is a good framework for understanding and modeling an ideal financial market, but provides only an approximate description of real financial markets.

\subsection{The Stratonovich convention}

In general a stochastic differential equation (SDE) driven by state-dependent white noise, like a geometric Brownian motion for example, is not coherent unless the multiplicative white noise term is well defined. There are two different interpretations that are well known, the Ito ${ }^{49}$ and the Stratonovich. ${ }^{50}$ The Ito formulation is commonly preferred by mathematicians while the Stratonovich interpretation mostly favored amongst physicists.

The interpretation of a stochastic differential equation arises when dealing with a multiplicative stochastic differential equation, also called a multiplicative Langevin equation

$$
\frac{d Y}{d t}=f(Y)+g(Y) \epsilon(t)
$$

where $f$ and $g$ are given functions and $\epsilon(t)$ is the Gaussian white noise, that is, a Gaussian and stationary random process with zero mean and delta correlated. Alternatively Eq. (18) may be written in terms of the Wiener process $W(t)$, as in Eq. (1), where $d W(t)=\epsilon(t) d t$. When $g$ depends on $Y$, Eq. (18) and Eq. (1) have no meaning unless an interpretation of the multiplicative term $g(Y) \epsilon(t)$ is provided, this interpretation must be given because, due to the extreme randomness of white noise.

The value that $Y$ should take is not exactly obvious even during an infinitesimal timestep $d t$. As we saw in Section 2.2, for an Ito process, the value for $Y$ is defined as $Y \equiv Y(t)$. Alternatively the value for $Y$ may be defined at the middle of the time step

$$
Y \equiv Y\left(t+\frac{d t}{2}\right)=Y(t)+\frac{1}{2} d Y(t) .
$$

This is known as the Stratonovich convention. 
The interpretation chosen will mainly make a difference when one is considering the product of two random processes, this is because the resulting differential will adopt a different expression as a result. The differential of the product of two random processes is defined as

$$
d(X Y)=[(X+d X)(Y+d Y)]-X Y .
$$

This may be written

$$
d(X Y)=X d X+Y d Y+d X d Y
$$

or equivalently one may write the product of differential as

$$
d(X Y)=\left(X+\frac{d X}{2}\right) d Y+\left(Y+\frac{d Y}{2}\right) d X .
$$

We say that the differential of a product reads in the Stratonovich interpretation when

$$
d(X Y)=X_{S} d Y+Y_{S} d X
$$

where $Y_{S}$ and $X_{S}$ are defined by Eq. (19).

In contrast we say that the differential of a product follows the Ito interpretation when

$$
d(X Y)=X_{I} d Y+Y_{I} d X+d X d Y,
$$

where $X_{I}(t)=X(t)$ and similarly for $Y(t)$. Equations (22) and (24) can be generalized as the product of two functions, $\omega(Y)$ and $\xi(Y)$ for example. In this case we may rewrite, in the Stratonovich interpretation, Eq. (23) as

$$
d(\omega \xi)=\omega\left(Y_{S}\right) d \xi(Y)+\xi\left(Y_{S}\right) d \omega(Y),
$$

with $d \omega=\omega(Y+d Y)-\omega(Y)$, similarly for $d \xi(Y)$. One could also define a similar expression in terms of $\omega(Y)$ and $\xi(Y)$ for the Ito interpretation, namely

$$
d(\omega \xi)=\omega(Y) d \xi(Y)+\xi(Y) d \omega(Y)+d \omega(Y) d \xi(Y) .
$$

If we now go back to the Langevin equation, Eq. (18), we can see what are the consequences of the above result. In an Ito interpretation the value of $Y$, hence the value of $g(Y)$, describes a random process. That means that we have $\langle g(Y) \epsilon(t)\rangle=0$, in other words $g(Y)$ is independent of $\epsilon(t)$. On the other hand, it can be proved that within the Stratonovich framework the average of the multiplicative term reads $\left(g(Y) g^{\prime}(Y) / 2\right)$, see $\operatorname{Refs}^{51,52}$ for more details.

The important question is, how does one relate the SDE from one framework to the other. In the Stratonovich framework an SDE defined as in Eq. (1) can be written as

$$
d Y=f^{(S)}\left(Y_{S}\right) d t+g^{(S)}\left(Y_{S}\right) d W(t) .
$$

where $Y_{S}$ is defined in Eq. (19). In the Ito convention we have

$$
d Y=f^{(I)}\left(Y_{I}\right) d t+g^{(I)}\left(Y_{I}\right) d W(t) .
$$

The functions $f^{(S)}$ and $f^{(I)}$ are different functions and may be related in the following ${ }^{52}$

$$
f^{(I)}(Y)=f^{(S)}(Y)-\frac{1}{2} g^{(S)}(Y) \frac{\partial g^{(S)}(Y)}{\partial Y}
$$

while the relation between the multiplicative functions $g^{(S)}$ and $g^{(I)}$ is defined as

$$
g^{(S)}(Y)=g^{(I)}(Y)
$$


Another crucial difference between the Ito and Stratonovich interpretation appears to be when a change of variables is made on the original equation. It can be proved that when the Stratonovich convention is used the standard rules of calculus hold as opposed to the Ito convention where new rules start to appear.

Let $h(Y, t)$ be an arbitrary function of $Y$ and $t$. In the Ito sense, the differencial of $h(Y, t)$ reads $^{52}$

$$
d h(Y, t)=\frac{\partial h(Y, t)}{\partial Y} d Y+\left[\frac{\partial h(Y, t)}{\partial t}+\frac{1}{2} g^{2}(Y, t) \frac{\partial^{2} h(Y, t)}{\partial Y^{2}}\right] d t
$$

whereas in the Stratonovich sense, we have

$$
d h(Y, t)=\frac{\partial h\left(Y_{S}, t\right)}{\partial Y_{S}} d X+\frac{\partial h\left(Y_{S}, t\right)}{\partial t} d t
$$

Inserting Eq. (28) into Eq. (31) one recovers Ito's lemma defined in Appendix A, that is extensively used in mathematical finance books.

\section{THE PATH INTEGRAL APPROACH: AN INTRODUCTION}

The path integral method is an integral formulation of the dynamics of a stochastic process. The method becomes useful when one wants to calculate the transition probabilities, which are associated to a given stochastic process. In this framework finite time transition probability can be written as a convolution of short time transition probabilities $^{36,38,44,45}$

Once an explicit method is written down to calculate the transitions probabilities it is possible to calculate the expectation values of the quantities of financial interest, given by the form

$$
E\left[\theta_{i} \mid Y_{i-1}\right]=\int d z_{i} p\left(z_{i} \mid z_{i-1}\right) \theta_{i}\left(e^{z_{i}}\right)
$$

where $z=\ln Y, p\left(z_{i} \mid z_{i-1}\right)$ is the transition probability, $\theta_{i}$ is an option at a given time slice $t_{i}$ and $Y_{i}$ is the price of an asset at a given time slice $t_{i}$. In this case $E\left[\theta_{i} \mid Y_{i-1}\right]$ is the conditional expectation value of some functional $\theta_{i}$ of the stochastic process. i.e.,

The probability distribution function related to SDE verifies the so called Chapman-Kolmogorov equation, ${ }^{41}$

$$
p\left(z^{\prime \prime} \mid z^{\prime}\right)=\int d z p\left(z^{\prime \prime} \mid z\right) p\left(z \mid z^{\prime}\right),
$$

which can be interpreted as the probability density of a transition from the value $z^{\prime}$ at time $t^{\prime}$ to the value $z^{\prime \prime}$ at time $t^{\prime \prime}$ summed over all possible intermediate values $z$ of the probability of separate and consequent transitions $z^{\prime} \rightarrow z$ and $z \rightarrow z^{\prime \prime}$. If we now consider a finite time interval $\left[t^{\prime}, t^{\prime \prime}\right]$ and we apply a time slicing, by considering $n+1$ subintervals of length $\Delta t \equiv\left(t^{\prime \prime}-t^{\prime}\right) / n+1$ then Eq. (34) becomes

$$
p\left(z^{\prime \prime} \mid z^{\prime}\right)=\int_{-\infty}^{+\infty} \cdots \int_{-\infty}^{+\infty} d z_{1} \cdots d z_{n} p\left(z^{\prime \prime} \mid z_{n}\right) p\left(z_{n} \mid z_{n-1}\right) \cdots p\left(z_{1} \mid z^{\prime}\right) .
$$

Now based on some realistic assumptions one needs to write down an expression for the transition probabilities $p\left(z_{i} \mid z_{i-1}\right)$ and then perform the calculation of the path integrals.

\subsection{Transition probabilities with a Gaussian}

In general we do not have an explicit expression for the transition probabilities for small time steps, however using a Gaussian transition probability ${ }^{38,44,45}$

$$
p\left(z^{\prime} \mid z\right)=\frac{1}{\sqrt{2 \pi \sigma^{2} \Delta t}} \exp \left\{-\frac{\left(z^{\prime}-z\right)^{2}}{2 \sigma^{2} \Delta t}\right\},
$$


where $\Delta t=t^{\prime}-t$, a general expression of the transition probability for small time steps correct up to $\mathcal{O}(\Delta t)$ is given by ${ }^{44}$

$$
p\left(z^{\prime} \mid z\right)=\frac{1}{\sqrt{2 \pi \sigma^{2}(z) \Delta t}} \exp \left\{-\frac{\left[z^{\prime}-z-A(z) \Delta t\right]^{2}}{2 \sigma^{2}(z) \Delta t}\right\}
$$

Eq. (37) gives a prescription to write the solution of a Fokker-Planck equation in the form of a convolution product of short-time transition probability functions. Substituting Eq. (37) into Eq. (35) we obtain

$$
\begin{aligned}
p\left(z^{\prime \prime} \mid z^{\prime}\right) & =\int_{-\infty}^{+\infty} \cdots \int_{-\infty}^{+\infty} d z_{1} \cdots d z_{n} \prod_{k=1}^{n+1} \frac{1}{\sqrt{2 \pi \sigma^{2}(z) \Delta t}} \exp \left\{-\frac{\left[z_{k}^{\prime}-z_{k-1}-A(z) \Delta t\right]^{2}}{2 \sigma^{2}(z) \Delta t}\right\} \\
& =\int_{-\infty}^{+\infty} \cdots \int_{-\infty}^{+\infty} d z_{1} \cdots d z_{n} \frac{1}{\sqrt{\left(2 \pi \sigma^{2}(z) \Delta t\right)^{n+1}}} \exp \left\{-\frac{1}{2 \sigma^{2}(z) \Delta t} \sum_{k=1}^{n+1}\left[z_{k}^{\prime}-z_{k-1}-A(z) \Delta t\right]^{2}\right\}
\end{aligned}
$$

Now if we set

$$
S_{0}\left(z_{k} ; z_{k-1}\right)=\frac{1}{2 \sigma^{2}(z)}\left[\frac{\left(z_{k}-z_{k-1}\right)}{\Delta t}-A\right]^{2} \Delta t,
$$

note that the term $\left(z_{k}-z_{k-1}\right) / \Delta t$ in Eq. (40) can be interpreted as a a mean velocity in the time interval $\Delta t$ and $1 / \sigma^{2}$ as a mass, then Eq. (38) may be written as

$$
p\left(z^{\prime \prime} \mid z^{\prime}\right)=\int_{-\infty}^{+\infty} \cdots \int_{-\infty}^{+\infty} d z_{1} \cdots d z_{n} \prod_{k=1}^{n+1} \frac{1}{\sqrt{2 \pi \sigma^{2}(z) \Delta t}} \exp \left\{-S_{0}\left(z_{k} ; z_{k-1}\right)\right\} .
$$

Then a Lagrangian structure appears. Hence in the limit as $n \rightarrow \infty$, Eq. (41) may be formally written as the path integral

$$
p\left(z^{\prime \prime} \mid z^{\prime}\right)=\int_{\tilde{z}(t)=z}^{\tilde{z}\left(t^{\prime}\right)=z^{\prime}} \mathcal{D}\left[\sigma^{-1} \tilde{z}\right] \exp \left\{-\int_{t}^{t^{\prime}} L_{0}[\tilde{z}(\tau) ; \dot{\tilde{z}}(\tau) ; \tau] d \tau\right\} .
$$

The functional measure, $\mathcal{D}\left[\sigma^{-1} \tilde{z}\right]$, means summations on all possible paths starting from $z^{\prime}$ and arriving at $z$. The integral to the exponent must be interpreted as the limit of the discrete summation for $n \rightarrow \infty$ and the function $L_{0}[\tilde{z}(\tau) ; \dot{\tilde{z}}(\tau) ; \tau]$ is a Lagrangian function defined by

$$
L_{0}[\tilde{z}(\tau) ; \dot{\tilde{z}}(\tau) ; \tau]=\frac{1}{2 \sigma^{2}}[\dot{\tilde{z}}-A(z, \tau)]^{2} .
$$

Since in the limit $n \rightarrow \infty$ only $\mathcal{O}(\Delta t)$ contribute to the integral, the formal expression in Eq. (42) gives the exact finite transition probability, moreover there is a complete equivalence between the differential stochastic equation, the Fokker-Planck equation and the path integral approach. ${ }^{44}$

This formalism is used to calculate the transition probabilities. Once these are evaluated the option price is computed as the conditional expectation value of a given functional of the stochastic process. For example, the price of an European call option will be given by

$$
\mathcal{C}=\exp \left[-r\left(t^{\prime}-t\right)\right] \int_{-\infty}^{+\infty} d z^{\prime} p\left(z^{\prime}, t^{\prime} \mid z, t\right) \max \left[e^{z^{\prime}}-X, 0\right],
$$

with $X$ being the strike price. While for an European put, it will be

$$
\mathcal{P}=\exp \left[-r\left(t^{\prime}-t\right)\right] \int_{-\infty}^{+\infty} d z^{\prime} p\left(z^{\prime}, t^{\prime} \mid z, t\right) \max \left[X-e^{z^{\prime}}, 0\right],
$$


where $r$ is the risk-free interest rates.

The method may also be applied to American options and can be extended to cope with other financial derivatives (with path dependent features). This formalism can be extended to other models beyond the dynamics of geometric Brownian motion. In Ref., ${ }^{38}$ Montagna et. al. have used this formalism to compare their results with those available in the literature for European and American options in the Black-Scholes model and found to be in good agreement with the standard numerical procedures used in finance.

Other applications of path integrals may be found in previous works. Linetsky ${ }^{46}$ who showed how path dependent options could be formally priced in a path integral framework. He considered several examples of one and two factor path dependent options. Baaquie ${ }^{53}$ has shown how path integrals methods can be used to price vanilla options with stochastic volatility. In a subsequent publication the same author has also shown how the Heath-Jarrow-Morton (HJM) formulation of treasury bonds in terms of forward rates can be reformulated as a problem in path integration. ${ }^{54}$ Later on Otto $^{21}$ demonstrated how to use path integration to price bonds and bond options under general short rate models.

\section{AN EXAMPLE WITH MAXIMAL ENTROPY}

In this section we describe a small mode ${ }^{55}$ that describes the evolution of money distribution from a Gaussian to a non-Gaussian distribution. These types of distribution are sometimes referred as a fat tail distribution.

In a series of lectures, ${ }^{56}$ Carter presented a simple agent based economic model. In this model every agent randomly selects someone else among a collection of agents and gives to the target one dollar until he runs out of money. Carter and Shapiro showed, using maximal entropy, that no matter how the money is initially distributed ultimately it obeys a Boltzmann-Gibbs distribution.

The model is constructed on a series of events or more precisely a series of transactions. The amount given out by every agent at a transaction is $\Delta x$ in some scaled units. It is possible that one agent could be visited by many agents. Supposing that one adopts the following notation: $N$ is the total number of agents, $M$ is the total amount of money involved and $n_{x}(t)$ is the expected number of agents with money $x$ and time $t$.

In this case we have the following events for a particular time $t$

$$
\begin{aligned}
E_{n_{x}} & =. N O T .\{x \text { agent visited only by one non }- \text { zero agent }\} \\
E_{n_{x+\Delta x}} & =\{(x+\Delta x) \text { agent visited only by zero non }- \text { zero agent }\} \\
E_{n_{x-\Delta x}} & =\{(x-\Delta x) \text { agent visited only by two non }- \text { zero agent }\} \\
E_{n_{x-2 \Delta x}} & =\{(x-2 \Delta x) \text { agent visited only by three non }- \text { zero agent }\} \\
& \cdots \\
E_{n_{\Delta x}} & =\left\{\Delta x \text { agent visited only by } \frac{x}{\Delta x} \text { non }- \text { zero agent }\right\} \\
E_{n_{0}} & \left.=\text { zero agent visited only by } \frac{x}{\Delta x} \text { non }- \text { zero agent }\right\}
\end{aligned}
$$

The increase in expected number of $x$-agents are then

$$
n_{x}(t+\Delta t)-n_{x}(t)=-n_{x} P\left(E_{n_{x}}\right)+n_{x+\Delta x} P\left(E_{n_{x+\Delta x}}\right)+\cdots+n_{\Delta x} P\left(E_{n_{\Delta x}}\right)+n_{0} P\left(E_{n_{0}}\right)
$$

where $P_{k}=P($ an agent visited by $k$ non-zero agent) is the probability associated with the respective events. X.S. Liang and T. Carter ${ }^{56}$ have chosen to use a binomial distribution

$$
P_{k}= \begin{cases}C_{N-n_{0}-1}^{k} q^{k}(1-q)^{N-n_{0}-1-k} & \text { for } k \leq N-n_{0}-1 \\ 0 & \text { otherwise. }\end{cases}
$$

where $q=1 /(N-1)$. Then the probabilities in Eq. (47) can be evaluated as

$$
\begin{aligned}
P\left(E_{n_{x}}\right) & =1-P_{1}, \\
P\left(E_{n_{x+\Delta x}}\right) & =P_{0},
\end{aligned}
$$




$$
\begin{aligned}
P\left(E_{n_{x-\Delta x}}\right) & =P_{2}, \\
P\left(E_{n_{x-2 \Delta x}}\right) & =P_{3}, \\
& \cdots \\
P\left(E_{n_{\Delta x}}\right) & =P_{x / \Delta x}, \\
P\left(E_{n_{0}}\right) & =P_{x / \Delta x} .
\end{aligned}
$$

It implies that Eq. (47) may be rewritten as

$$
n_{x}(t+\Delta t)-n_{x}(t)=-n_{x}+\left[\sum_{k=0}^{x / \Delta x} n_{x+(1-k) \Delta x} P_{k}\right]+n_{0} P_{x / \Delta x} .
$$

Normalizing $n_{x}$ with the total number of agents $N$, they obtain the probability assigned to money $x$. The coefficients of each probability are the probability density functions (pdf). Hence, the equation governing the evolution of the pdf of money $x$ at time $t$ is defined as

$$
f(x, t+\Delta t)=\sum_{k=0}^{x / \Delta x} f(x+(1-k) \Delta x, t) P_{k}+f(0, t) P_{x / \Delta x}, \quad \text { given that } \mathrm{x} \in[0, \mathrm{M}] .
$$

In this formulation the total number of agents $N$ and the total number money $M$ are preserved. In terms of $f$, the following two conservation laws as expressed as

$$
\begin{aligned}
& \int_{0}^{M} f(x, t) d x=1 \quad \text { conservation of } \mathrm{N}, \\
& \int_{0}^{M} f(x, t) d x=\frac{M}{N} \text { conservation of } \mathrm{M} .
\end{aligned}
$$

The probability space of Eq. (51) is not the whole real line. Therefore the boundary conditions need to be set for $x=0$ and $x=M$. If we follow the arguments in Ref., ${ }^{56}$ the left boundary condition is set to

$$
f(0, t+\Delta t)=[f(0, t)+f(\delta x, t)] \exp (-1+f(0, t) \Delta x),
$$

and at the right boundary $x=M, f$ can only have two choices: $1 / N \Delta x$ and 0 . The former is unstable as one transaction will revoke the membership of the $M$-agent. Hence it is set as $f(M, t)=0$.

In the asymptotic region Eq. (51) may be simplified to an advection-diffusion equation. In general the system takes a long time to reach equilibrium, or its steady distribution. If by any chance, the money distribution is away from the origin, the advection halts and the solution of $f$ adopts a form of Gaussian. Gaussian dominates the pdf evolution before it hits the left boundary. The evolution of the system starts as a Gaussian distribution and when the tail of the Gaussian hits the left boundary at $x=0$, then the whole structure starts to drift leftwards and gives itself away to other structures.

In this model it can be shown that the steady state or equilibrium solution is the same as the Boltzmann solution obtained from the maximal entropy principle. ${ }^{56}$ This condition does not hold near the origin. To learn more about the steady state probability distribution near the origin one needs to use the boundary conditions. It is found that the numbers of zero agents and $\Delta x$ agent take a finite value and do not follow the Boltzmann distribution in that region.

A numerical simulation of Eq. (51), using Monte Carlo, shows that the evolution of the system first resembles a Gaussian like shape centered at a fixed value. This state lasts for a considerable amount of time, even after it hits the left boundary. The system then evolves towards an exponential similar to a Boltzmann distribution (or an exponential decay). 


\section{APPROACH}

Our approach in calculating the required transition probabilities, for insertion into the path integrals, is to divide a time interval into infinitesimal segments of a given length, then consider all possible paths starting at a given point in that space at an initial time and ending at a later point in that space at a final time. Each path is then approximated by straight line segments in each infinitesimal time interval and is weighted by some exponential factor that is directly related to the payoff at a given expiration at a time $T$. The payoff is equal to a given function of the terminal asset price $S_{T}$. It is the expected value over the risk neutral measure conditional on the initial price $S$ at time $t$. This average can be represented as an integral over the set of all paths originating from a time $t$ and a price $S$. This is what we call the path integral. This path integral can take various forms depending on the structure of the option taken into consideration, i.e., a path dependent or not option. However in both cases the path integral is similar. Now if we consider the Black-Scholes equation for example, we see that the Black-Scholes action functional defined on paths as a time integral of the Black-Scholes Lagrangian function appears in the path integral and acts as a path integration measure. In this case the Black-Scholes Lagrangian is really a functional that relates the backward Kolmogorov equation for the risk-neutral diffusion process with drift rate equal to the risk free rate to the action. In other words the Lagrangian is a functional that relates the stochastic differential equation for the financial system under scrutiny to the action functional. It can also be thought of as the transition probability density function, which acts as a probability on each path. This determines which paths will make a contribution to the expectation value, and hence the payoff. In the case of multi-asset derivatives with a general multi- Dimensional diffusion process only the action functional differs. In fact, it is only the Lagrangian functional, which differs depending on which option one considers. ${ }^{46}$ This action functional is then summed over all possible values of the parameter space at intermediate times and finally normalized with a factor that is proportional to the number of degrees of freedom of the system. Then we will take the analytical limit of the interval length to zero and the number of intervals to infinity while keeping the difference between the final and initial time fixed.

The approach here will be to use path integral techniques to find an accurate way of calculating the option price. The general approach will be to work backwards and start to tackle the problem from the end of the problem. That is, using existing data to construct a basic numerical model governed by a set of relevant fundamental parameters as input data for the path integral. This method may be repeated recursively.

The data that we will use are Eurodollars. Eurodollars are fixed time deposits held primarily by overseas banks, but denominated in a US dollar denomination. They are not subject to US banking regulations and therefore tend to have a tighter bid-ask spread than deposits held in the United States. ${ }^{57}$ The 3-month Eurodollar future is one of the most actively traded futures markets in the world and is quoted as an index where the yield is equal to the Eurodollar price subtracted from 100. This yield is equal to the fixed rate of interest paid by the Eurodollar time deposits upon maturity and is expressed as an annualised interest rate based on a 360-day year. The Eurodollar futures are cash settled based on the 90-day London Interbank Offer Rate (LIBOR). A national principal amount of 1 million, is used to determine the change in the total interest payable on a hypothetical underlying time deposit, but is never actually paid or received. ${ }^{57}$

The options traded on the Eurodollar futures include not only 18 months of options expiring at the same time as the underlying future, but also various short dated options which themselves expire up to 1 year prior to the expiration of the underlying futures contract. In this project, the approach builds upon the ideas presented in one of our papers. ${ }^{58}$

The construction of the transition probability will be done using real data. The approach to extract a model for the probability transition will be done using stochastic calculus, incorporating stochastic volatility. ${ }^{42,59}$ In the case of the stochastic volatility market model one can see that there is a whole family of equivalent martingale measures, and derivatives securities cannot be perfectly hedged with just the stock and bond. This is what is called incomplete markets, oppositely complete markets are those where there is a unique equivalent martingale measure under which the discounted prices of traded securities are martingale. As a result incomplete market models will also be taken into consideration. ${ }^{42}$ As a first approach, we will treat the system as a Markov process, and we will use the techniques and methods for extracting the transition probabilities described in a book written by S.P. Meyn and R.L. Tweedie on Markov chains and stochastic stability. ${ }^{60}$ This aspect of the model formulation varies for each different system because it is clear that each system typically presents its own 
non-typical unique character and this must be included in any such analysis. This is why working with real data to extract the transition probabilities for each system in question is of crucial importance to provide a general solution for each system.

The numerical approach will be done using techniques known in numerical integration of stochastic differential equations and techniques known to the integration of path integrals ${ }^{61}$ and will be compared to other option pricing model results. ${ }^{62}$ In the Nag program library ${ }^{63}$ there is a vast array of numerical methods to perform numerical integration, in a similar way Monte Carlo methods can be adopted to perform the integration as it is outlined in. ${ }^{45}$ For the integration there is also a robust and accurate histogram-based (non-Monte Carlo) path integral algorithm to calculate the long time probability distribution that has been developed to handle non-linear Lagrangians. ${ }^{64-70}$ This histogram procedure recognizes that the distribution can be numerically approximated to a high degree of accuracy as a sum of rectangles at given points of a given height and with a given width ${ }^{39}$.

For calculation of the option, only one-dimensional integrals are needed and are simply performed with a standard trapezoidal rule, which can be cross checked with the adaptive integration DO1EAF routine from the Nag Library. ${ }^{63}$

For the optimising the size of the window, some calibration work will be required. The size of the window is directly proportional to the size of the data file taken into consideration. We will test various window sizes first and then use a Genetic Algorithm, for example ${ }^{71}$ to select an optimal window from a large sample set of window sizes. For the boundary conditions of the problem these should become clear from the determination of the window size and from the calculation of the path integral.

\section{CONCLUSION}

In this short review we introduced the idea of using path integrals to further explore the fluctuations in financial markets. We also gave a brief description of the Black-Scholes equation for idealized markets. This review is intended to be an introduction to econoenginnering and will be used in our future work.

\section{APPENDIX A. ITO'S LEMMA}

The price of a stock option is a function of the underlying stock's price $Y(t)$ and time $t$. More generally, we can say that the price of any derivative security is a function $G$, of the stochastic variables underlying the derivative security and time. In 1951 a mathematician called Ito, discovered an important result, known as Ito's Lemma. ${ }^{49}$

If we consider a continuous and differentiable function $G$ of a variable $x$, i.e., $G(x)$, and if $\Delta x$ is a small change in $x$ which leads to $\Delta G$, a small change in $G$, then it is well known that,

$$
\Delta G=\frac{d G}{d x} \Delta x+\mathcal{O}\left(\Delta x^{2}\right)
$$

i.e., $\Delta G$ is approximately equal to the rate of change of $G$ with respect to the variable $x$ times $\Delta x$. More precisely a Taylor expansion of $\Delta G$ will tell us that

$$
\Delta G=\frac{d G}{d x} \Delta x+\frac{1}{2} \frac{d^{2} G}{d x^{2}} \Delta x^{2}+\frac{1}{6} \frac{d^{3} G}{d x^{3}} \Delta x^{3} \cdots
$$

In the case $G$ is a continuous differentiable function of two variables, $G(x, y)$,

$$
\Delta G(x, y)=\frac{\partial G(x, y)}{\partial x} \Delta x+\frac{\partial G(x, y)}{\partial y} \Delta y+\mathcal{O}\left(\Delta x^{2}, \Delta y^{2}\right)
$$

which means that the Taylor expansion of $\Delta G$ is then

$$
\Delta G=\frac{\partial G}{\partial x} \Delta x+\frac{\partial G}{\partial y} \Delta y+\frac{1}{2} \frac{\partial^{2} G}{\partial x^{2}} \Delta x^{2}+\frac{\partial^{2} G}{\partial x \partial y} \Delta x \Delta y+\frac{1}{2} \frac{\partial^{2} G}{\partial y^{2}} \Delta y^{2} \cdots
$$


In the limit of $\Delta x, \Delta y \rightarrow 0$, Eq. (58) becomes

$$
d G=\frac{\partial G}{\partial x} d x+\frac{\partial G}{\partial y} \Delta y
$$

A derivative security is a function of a variable that follows a stochastic process. Supposing that a variable $x$ follows the general Ito process ${ }^{\dagger}$

$$
d x=a(x, t) d t+b(x, t) d W,
$$

and that $G$ is also a function of $x$ and $t$. Hence from Eq. (58),

$$
\Delta G=\frac{\partial G}{\partial x} \Delta x+\frac{\partial G}{\partial t} \Delta t+\frac{1}{2} \frac{\partial^{2} G}{\partial x^{2}} \Delta x^{2}+\frac{\partial^{2} G}{\partial x \partial t} \Delta x \Delta t+\frac{1}{2} \frac{\partial^{2} G}{\partial t^{2}} \Delta t^{2} \cdots .
$$

A Wiener process is a particular type of Markov stochastic process. The behavior of a variable $W$ which follows a Wiener process can be understood by considering the changes in its value in small intervals of time $t$. If we consider a small interval of time of length $\Delta t$ and if we define $\Delta W$ as the change in $W$ during $\Delta t$, then there are two basic properties of $\Delta W$ :

Property 1. $\Delta W$ is related to $\Delta t$ by the equation $\Delta W=\epsilon \sqrt{\Delta t}$, where $\epsilon$ is a random sample from a standardized normal distribution (mean of $\Delta W=0$, standard deviation of $\Delta W=\sqrt{\Delta t}$, and the variance of $\Delta W=\Delta t$ ).

Property 2. The values of $\Delta W$ for any two different short intervals of time $\Delta t$ are independent (This implies that $W$ follows a Markov process).

Now because an Ito process is a generalized version of the Wiener process, properties $\mathbf{1}$ and $\mathbf{2}$. also holds for an Ito process. Hence a discretized version of Eq. (60) takes the form

$$
\Delta x=a(x, t) \Delta t+b(x, t) \epsilon \sqrt{\Delta t},
$$

which implies that

$$
\Delta x=\epsilon^{2} b^{2}(x, t) \Delta t+\text { higher order terms in } \Delta \mathrm{t} .
$$

This shows that the term involving $\Delta x^{2}$ in Eq. (61) has components that is of order $\Delta t$ and cannot be ignored, unlike with Eq. (58) when the limit $\Delta x, \Delta y \rightarrow 0$ was taken to obtain Eq. (59).

The variance of a standardized normal distribution is 1 , this means that

$$
E\left(\epsilon^{2}\right)-[E(\epsilon)]^{2}=1
$$

where $E$ is the expectation value. Since $E(\epsilon)=0$, it follows that $E\left(\epsilon^{2}\right)=1$. The expected value of $\epsilon^{2} \Delta t$ is therefore $\Delta t$. It can be shown that the variance of $\epsilon^{2} \Delta t$ is of the order of $\Delta t$, and that as a results of this, $\epsilon^{2} \Delta t$ becomes non-stochastic and equals to its expected value of $\Delta t$ as $\Delta t \rightarrow 0$. Hence taking the limit as $\Delta x, \Delta t \rightarrow 0$ in Eq. (61) together with Eq. (63) we therefore obtain

$$
d G=\frac{\partial G}{\partial x} d x+\frac{\partial G}{\partial t} d t+\frac{1}{2} \frac{\partial^{2} G}{\partial x^{2}} b^{2} d t
$$

This is known as the Ito Lemma. Now substituting for $d x$ from Eq. (60), Eq. (65) becomes

$$
\begin{aligned}
d G & =\frac{\partial G}{\partial x}[a(x, t) d t+b(x, t) d W]+\frac{\partial G}{\partial t} d t+\frac{1}{2} \frac{\partial^{2} G}{\partial x^{2}} b^{2} d t \\
d G & =\left(\frac{\partial G}{\partial x} a(x, t)+\frac{\partial G}{\partial t}+\frac{1}{2} \frac{\partial^{2} G}{\partial x^{2}} b^{2}\right) d t+\frac{\partial G}{\partial x} b(x, t) d W .
\end{aligned}
$$

\footnotetext{
${ }^{\dagger}$ A Ito process is a generalized Wiener process $d x=a d t+b d W$ where the parameters $a$ and $b$ are functions of the underlying variable $x$ and time $t$.
} 


\section{REFERENCES}

1. A. Allison and D. Abbott, "Stable processes in an econometric time series," American Inst. Physics 511, pp. 221-232, 2000 .

2. F. Michael and M. Johnson, "Financial market dynamics," Physica A 320, pp. 525-534, 2003.

3. P. Buchen and M. Kelly, "The maximum entropy distribution of an asset inferred from option prices," The Journal of Finance and Quantitative Analysis 31 No. 1, pp. 143-159, 1996.

4. A. Krawieecki and J. Holyst, "Stochastic resonance as a model for financial market crashes and bubbles," Physica A 317, pp. 597-608, 2003.

5. E. E. Haven, "A discussion on embedding the black and scholes option pricing model in a quantum physics setting," Physica A 304, pp. 507-524, 2002.

6. D. Faller and F. Petruccione, "A master equation approach to option pricing," Physica A 319, pp. 519-534, 2003.

7. A. A. Stanislavsky, "Black-scholes model under subordination," Physica A 318, pp. 469-474, 2003.

8. L. Borland, "Option pricing formulas based on a non-gaussian stock price model," Phys. Rev. Lett. 89, p. 098701, 2002.

9. L. Borland, "A theory of non-gaussian option pricing," Quantitative Finance 2, pp. 415-431, 2002.

10. L. Borland, "Microscopic dynamics of the nonlinear fokker-planck equation: A phenomenological model," Phys. Rev E 57, p. 6634, 1998.

11. C. Tsallis, "Possible generalization of boltzmann-gibbs statistics," J. Stat. Phys. 52, p. 479, 1988.

12. L. Bachelier, Theorie de la speculation, L. Gobay, Sceaux, 1995 (reprinted in P. Cootner, Ed., the random character of stock market prices, MIT Press, Cambridge, MA, 1964, pp 17-78)., 1964.

13. A. Einstein, "Uber die von der molakular kinetisken theorie der warne geforderte bewegaung von in ruhenden flussigkeiten suspendierten teilchen," Ann. Phys. 17, p. 549, 1905.

14. N. Wiener, "Differential-space," J. Math. Phys. 2, p. 131, 1923.

15. F. Black and M. Scholes, "Equations of state calculations by fast computing machine," J. Pol. Econ. 81, p. $637,1973$.

16. R. C. Merton, "Theory of rational option pricing," Bell J. Econ. Management Sci 4, pp. 141-183, 1973.

17. J. Bouchaud and M. Potters, Theory of financial risks, from statistical physics to risk management, Cambridge University Press., Cambridge, 2000.

18. Y. Maleverne and D. Sornette, "Multi-moments method for portfolio management: Generalized capital asset pricing model in homogeneous and heterogeneous markets," pp. [hep--cond/0207475], 2003.

19. J. P. Bouchaud and D. Sornette, "The black-scholes option pricing problem in mathematical finance: Generalization and extensions for a large class of staochastic processes.," J.Phys.I France 4, pp. 863-881, 1994.

20. S. Fedotov and S. Mikhailov, "Elements for a theory of financial risks," available at http://xxx.lanl.gov, cond-mat/9806101.

21. M. Otto, "Using path integrals to price interest rate derivatives," available at http://xxx.lanl.gov, cond-mat/9812318, 1999.

22. N. Wiener, "The average of an analytic functional and brownian motion," Proc. Natl. Acad. Sci. (USA) 7, p. $253,1921$.

23. N. Wiener, "The average of an analytic functional," Proc. Natl. Acad. Sci. (USA) 7, p. 294, 1921.

24. R. P. Feynman, "Space-time approach to non-relativistic quantum mechanics," Rev. Mod. Phys. 20, p. 367, 1948.

25. F. D. Bonnet, P. O. Bowman, D. B. Leinweber, and A. G. Williams, "Infrared behavior of the gluon propagator on a large volume lattice," Phys. Rev. D 62, p. 051501, 2000.

26. F. D. Bonnet, P. O. Bowman, D. B. Leinweber, A. G. Williams, and J. M. Zanotti, "Infinite volume and continuum limits of the landau gauge gluon propagator," Phys. Rev. D 64, p. 034501, 2001.

27. F. Bonnet, P. O. Bowman, D. B. Leinweber, A. G. Williams, and J. bo Zhang, "Overlap quark propagator in landau gauge," Phys. Rev. D 65, p. 114503, 2002.

28. F. Bonnet, D. Leinweber, A. G. Williams, J. Zanotti, and J. Zhang, "Quark propagator in a covariant gauge," Nucl.Phys.Proc.Suppl. 109A, pp. 158-162, 2002. 
29. J. Zhang, P. O. Bowman, D. B. Leinweber, A. G. Williams, and F. Bonnet, "Scaling behaviour of the overlap quark propagator in landau gauge," Submitted to Phys. Rev. D hep-lat/0301018.

30. F. D. Bonnet, D. B. Leinweber, and A. G. Williams, "General algorithm for improved lattice actions on parallel computing architectures," J. Comput. Phys. 170, pp. 1-17, 2001.

31. F. Bonnet, P. Fitzhenry, D. B. Leinweber, M. R. Stanford, and A. G. Williams, "Calibration of smearing and cooling algorithms in su(3): color gauge theory," Phys. Rev. D 62, p. 094509, 2000.

32. F. Bonnet, D. B. Leinweber, A. G. Williams, and J. M. Zanotti, "Improved smoothing algorithms for lattice gauge theory," Phys. Rev. D 65, p. 114510, 2002.

33. F. Bonnet, R. G. Edwards, G. T. Fleming, R. Lewis, and D. G. Richards, "Mesonic form factors," pp. hep--lat/0312008.

34. K. Ilinski, "Physics of finance," pp. hep--lat/9710148.

35. C. J. Coleman, "Huygen's principle applied to radio wave propagation," Rad. Sci. 37, p. No. 6 1105, 2002.

36. L. S. Schulman, Techniques and applications of path integrals, Wiley, New York, 1981.

37. A. Matacz, "Path dependent option pricing: the path integral partial averaging method," available at http://xxx.lanl.gov, cond-mat/0005319, 2000.

38. G. Montagna, O. Nicrosini, and N. Moreni, "A path integral way to option pricing," Physica A 310, pp. $450-466,2002$.

39. L. Ingber, "High-resolution path-integral development of financial options," Physica A 283, pp. 529-558, 2000.

40. J. Hull, Option, futures, and other derivatives, 4th edn. Prentice Hall, Wiley, New Jersey, 2000.

41. W. Paul and J. Baschnagel, Stochastic processes: from physics to finance, Springer, New York, 2000.

42. J. Fouque, G. Papanicolaou, and K. R. Sircar, Derivative in financial markets with stochastic volatility, Cambridge University Press, Cambridge, 2000.

43. G. Montagna, M. Morelli, O. Nicrosini, P. Amato, and M. Farina, "Pricing derivatives by path integral and neural networks," Physica A 324, pp. 189-195, 2003.

44. M. Rosa-Clot and S. Taddei, "A path integral approach to derivative security pricing: I. Formalism and analytical results," to be published in International Journal of Theoretical and Applied Finance, available at http://xxx.lanl.gov, cond-mat/9901277 .

45. M. Rosa-Clot and S. Taddei, "A path integral approach to derivative security pricing: II. Numerical methods," submitted to International Journal of Theoretical and Applied Finance, available at http://xxx.lanl.gov, cond-mat/9901279 .

46. V. Linetsky, "The path integral approach to financial modeling and option pricing," Computational Economics 11, pp. 129-163, 1998.

47. M. Rubinstein, "Implied binomial trees," The journal of Finance 49 No. 3, pp. 771-818, 1994.

48. J. C. Jackwerth and M. Rubinstein, "Recovering probability distribution from option prices," The journal of Finance 51 No. 5, pp. 1611-1631, 1996.

49. K. Ito, "On stochastic differential equations," Mem. Amer. Math. Soc. 4, pp. 1-51, 1951.

50. R. L. Stratonovich SIAM J. Control 4, pp. 362-371, 1966.

51. M. M. J. Perello, J.M. Porra and J. Masoliver, "Black-Scholes option pricing within Ito and Stratonovich conventions," Physica A 278, pp. 260-274, 2000.

52. C. W. Gardiner, Handbook of stochastic methods, Springer, New York, 1983.

53. B. E. Baaquie, "A path integral approach to option pricing with stochastic volatility: some exact results," J. Phys. I France 7, pp. 1733-1753, 1997.

54. B. E. Baaquie, "Quantum field theory of treasury bonds," available at http://xxx.lanl.gov, cond-mat/9809199 .

55. X. S. Liang and T. Carter, "Evolution of money distribution in a simple economic model," unpublished preprints .

56. T. Carter and Shapiro, "An introduction to information theory and entropy," CSSS course handouts , pp. Santa Fe Institute, Santa Fe, New Mexico, 2003. 
57. F. R. Bank, Instrument of the money markets, 7th Edition, Federal Reserve Bank of Richmond, Richmond, VA, 1993Thomas Carter and Shapiro, 1993.

58. F. Bonnet, A. Allison, and D. Abbott, "Review of quantum path integrals in fluctuating markets," Proc. SPIE 5274, p. in press, 2004.

59. A. Mele and F. Fornari, Stochastic volatility in financial markets, crossing the bridge to continuous time, Kluwer Academic Publisher, 2000.

60. S. Meyn and R. Tweedie, Markov chains and stochastic stability, Springer-Verlag, London, 1993.

61. C. Chiarella, N. El-Hassan, and A. Kucera, "Evaluation of american option prices in a path integral framework using fourier-hermite series expansions," Journal of Economic Dynamics and Control 23, pp. 1387$1424,1999$.

62. C. Chiarella, M. Craddock, and N. El-Hassan, "Evaluation of american option prices in a path integral framework using fourier-hermite series expansions," Computational Economics 22, pp. 113-138, 2003.

63. N. F. Library, The Numerical Algorithm Group Ltd, Oxford.

64. L. Ingber, M. Wehner, G. Jabbour, and T. Barnhill, "Application of statistical mechanics methodology to term-structure bond-pricing models," Mathl. Comput. Modelling 15 (1), pp. 65-90, 1991.

65. L. Ingber, "Path-integral evolution of short-term memory," Phys. Rev. E 49 (5B), pp. 4652-4664, 1994.

66. L. Ingber, "High resolution path-integral calculation of short-term memory," Phys. Rev. E 51 (5), pp. 50745083, 1995.

67. L. Ingber, "Path integral evolution of multivariate systems with moderate noise," Phys. Rev. E 51 (2), pp. 1616-1619, 1995.

68. M. Wehner and W. Wolfer, "Numerical evaluation of path integral solutions to fokker-planck equations i," Phys. Rev. A 27, pp. 2663-2670, 1983.

69. M. Wehner and W. Wolfer, "Numerical evaluation of path integral solutions to fokker-planck equations ii," Phys. Rev. A 28, pp. 3003-3011, 1983.

70. M. Wehner and W. Wolfer, "Numerical evaluation of path integral solutions to fokker-planck equations ii," Phys. Rev. A 35, pp. 1795-1801, 1987.

71. A. J. O. L. Fogel and M. J. Walsh, Artificial intelligence through simulated evolution, Wiley, New York, 1966. 